\title{
Deformation Model for Thioredoxin Catalysis of Disulfide Bond Dissociation by Force
}

\author{
Yuriy V. Pereverzev and Oleg V. Prezhdo \\ Department of Chemistry, University of Washington, Seattle, WA 98195, USA \\ (Received 4 March 2009; accepted 5 May 2009; published online 16 May 2009)
}

\begin{abstract}
We develop a theoretical model describing the effect of applied force on the rate of enzymatic dissociation of disulfide bonds (Wiita, A. P. et al., Nature 450:124-127, 2007). The timescale characterizing the extension of the 8-domain protein chain, in which every domain contains a disulfide bond, exhibits anomalous force dependence: The extension time first increases and then decreases with increasing force. This type of force dependence indicates catch-binding and is under active investigation in a variety of systems. The disulfide system presents the first example of a chemical catch-bond. The key difference between the deformation model developed here and the two-pathway model used in the original publication is the bond-deformation term in the force dependence of the dissociation rate constant. The bond-deformation concept provides a different interpretation of the phenomenon. Rather than invoking a new dissociation pathway, which is difficult to rationalize for a simple $\mathrm{S}-\mathrm{S}$ bond, catch-binding is explained by a force-induced deformation in the protein system disfavoring bond dissociation by thioredoxin. The analysis of the experimental data is performed within the Michaelis-Menten kinetic mechanism of enzyme catalysis. A simplified version of the MichaelisMenten mechanism containing only four parameters is found to provide a quantitative description of the key features of the experimental data.
\end{abstract}

Keywords-Catch-binding, Disulfide bond, Thioredoxin, Receptor-ligand interaction, Force-induced deformation, Enzyme catalysis.

\section{INTRODUCTION}

Recently, Fernandez and co-workers published a series of articles ${ }^{15,25,29,30}$ reporting novel and unexpected results regarding the enzymatic dissociation of disulfide bonds subjected to external force. A number of different enzymes were investigated. In particular, Wiita et al..$^{30}$ focused on several types of thioredoxin (Trx). The studies were performed with a chain of eight repeat units of the 27th immunoglobulin-like domains

Address correspondence to Oleg V. Prezhdo, Department of Chemistry, University of Washington, Seattle, WA 98195, USA. Electronic mail: prezhdo@u.washington.edu
(I27) of cardiac titin. Each I27 domain consisted of 89 residues and contained a disulfide bond between the residues 32 and 75 . Application of a $165 \mathrm{pN}$ force for $400 \mathrm{~ms}$ caused partial unfolding of the 8-domain chain, exposing the disulfide bonds. Further unfolding of the chain was observed only in the presence of the Trx enzyme. Using forces within the $30-600 \mathrm{pN}$ range, Fernandez and co-workers observed saturation of the chain extension at about $4 \mathrm{~s}$. In the absence of Trx, the force alone was not able to break the disulfide bonds.

Surprisingly, the bond lifetime first grew and only later decreased with increasing force strength. Typically, one expects that force speeds up dissociation. The observed anomalous behavior of the bond lifetime identifies so-called catch-binding. The catch-slip binding transitions were discovered recently in a number of receptor-ligand biological complexes. ${ }^{2,3,7,8,10,17,18,21-24,26,27,32}$ The disulfide bond presents the first example of a chemical catch-bond, i.e., a system in which the increased lifetimes are associated with breaking a covalent bond.

Wiita et al. ${ }^{30}$ proposed several closely related models of the observed effect. The best description of the experimental data was provided by a model postulating two distinct dissociation pathways for the disulfide bond dissociation. The two-pathway description of the catch-slip behavior using a single bound state was first proposed in Pereverzev et al., ${ }^{21}$ which focused on the anomalous force dependence of the measured lifetimes of the P,L-selectin/PSGL-1 bonds. ${ }^{17,24}$ By containing an intermediate state the model of Wiita et al. ${ }^{30}$ belongs to the class of two-state models, ${ }^{8,16,28}$ and therefore, it is more complex than model of reference. ${ }^{21}$ Nevertheless, the Wiita model is very close to the two-pathway model ${ }^{21}$ both conceptually and mathematically. The two-pathway model with one bound state is the simplest model of the catch-slip and other ${ }^{13}$ phenomena, and its straightforward mathematics leads to many useful analytic results. ${ }^{19,20,23}$ One of the conceptual problems with the two-pathway model is the need for two distinct dissociation pathways that must point in opposite directions. It may be hard to visualize 
the two pathways, especially for such a simple bond as the disulfide.

An alternative interpretation of catch-binding was given in Pereverzev and Prezhdo, ${ }^{18}$ which used a single dissociation pathway and attributed bond stabilization to a force-induced deformation impeding dissociation of the receptor-ligand bond. The model successfully described the behavior of the P-selectins/ PSGL- ${ }^{17}$ and actin/myosin ${ }^{10}$ bonds, see Pereverzev and co-workers, ${ }^{18,22}$ respectively. Recent atomistic simulations ${ }^{9,16,27}$ as well as experimental data ${ }^{1}$ support the deformation interpretation of catch-binding by showing that the proteins forming the bonds undergo large-scale conformational changes involving the binding pocket. The deformation concept allowed us to rationalize not only the catch-behavior but also the large discrepancies between the bond dissociation rate constants obtained by extrapolating the finite force data to zero force and directly without force. ${ }^{18}$ Depending on the magnitude and sign of the bond deformation energy, we classified biological bonds as stiff or deformable and as favorably or unfavorably deformed.

The current work applies the bond-deformation concept $^{18}$ to rationalize the anomalous behavior of the lifetime of the disulfide bond. ${ }^{30}$ The paper is constructed as follows. The next section considers the Michaelis-Menten scheme of enzyme catalysis involving the reactant, product, and intermediate states. Explicit analytic expressions for the time-dependent populations of the three states are given. A general expression for the time associated with breaking of the disulfide bond within one I27 domain is obtained. This bond dissociation time is related to the time-dependent extension of the chain composed of I27 domains. After that, the Michaelis-Menten scheme is analyzed for the experimental conditions of Wiita et $a l .{ }^{30}$ and an approximate solution to the full kinetic equations is derived. Then, the bond deformation concept ${ }^{18}$ is applied to the force-induced enzymatic dissociation of the disulfide bond, and an explicit force dependence of the main reaction rate is proposed. The theoretical results from the previous sections are applied to the experimental data, and the parameters of the deformation model of the enzymatic dissociation of the disulfide bond are established. The final section summarizes the key findings of our work.

\section{MICHAELIS-MENTEN MODEL OF ENZYME CATALYZED UNFOLDING OF A MULTIDOMAIN CHAIN}

The unfolding of the 8-domain protein chain is determined by whether or not the disulfide bonds

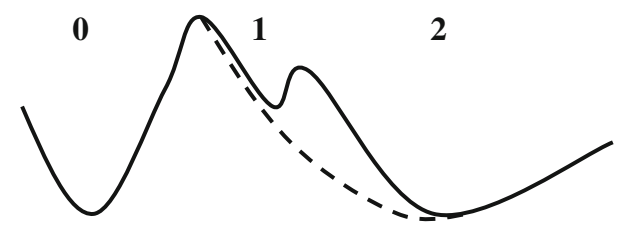

FIGURE 1. The energy profile of the reaction coordinate, solid line. Initially, state 0 , the disulfide pair and the enzyme are not interacting. State 1 is the intermediate state in the Michaelis-Menten mechanism. The disulfide pair is aligned with the groove of the enzyme. State 2 is the final state with broken disulfide bond. The dashed line shows a simplified energy profile, in which state 1 is short lived, and the system transitions from state 0 directly into state 2 .

contained in each domain have been broken by the applied force. The dissociation of the disulfide bonds is catalyzed by enzymes. To describe the probability of a disulfide bond to be broken by time $t$, we will use the classic Michaelis-Menten scheme of enzyme catalysis. The scheme includes three states. The initial state 0 involves an intact disulfide bond and an enzyme that exists in solution independently of the bond. The intermediate state 1 describes the bound disulfidebond/enzyme pair. The bond is appropriately aligned and weakened by interaction with the enzyme. The final state describes a broken disulfide bond. The energy profile including the three states separated by barriers along the reaction coordinate is depicted in Fig. 1. The intermediate state is shown in Fig. 1 as weakly bound, in agreement with the analysis of the experimental data performed in the next section.

Let $p_{0}(t), p_{1}(t)$, and $p_{2}(t)$ denote the probabilities of the disulfide pair to exist at time $t$ in state 0,1 , and 2, respectively. Since the bond must exist in one of the three states, the sum of the probabilities is equal to one

$$
p_{0}(t)+p_{1}(t)+p_{2}(t)=1 .
$$

The probabilities evolve according to the following system of kinetic equations

$$
\begin{aligned}
& \frac{d p_{0}(t)}{d t}=-k_{01} c p_{0}(t)+k_{10} p_{1}(t) \\
& \frac{d p_{1}(t)}{d t}=k_{01} c p_{0}(t)-\left(k_{10}+k_{12}\right) p_{1}(t) \\
& \frac{d p_{2}(t)}{d t}=k_{12} p_{1}(t)
\end{aligned}
$$

Here, $k_{i j}$ denotes transition rates between states $i$ and $j$, and $c$ is the enzyme concentration. The force dependence of the rate constants used in Eq. (2) is not yet specified. It will be introduced below following the analysis of the general solution of Eq. (2).

Considering that initially, at $t=0$ the probabilities are equal to $p_{0}(0)=1, p_{1}(0)=0$, and $p_{2}(0)=0$, the solution of Eq. (2) can be expressed as 


$$
\begin{aligned}
& p_{2}(t)=1+C_{1} \exp \left(-K_{1} t\right)+C_{2} \exp \left(-K_{2} t\right), \\
& p_{1}(t)=\frac{1}{k_{12}} \frac{d p_{2}(t)}{d t},
\end{aligned}
$$

where $K_{1}=\left(a_{1}-\sqrt{a_{1}^{2}-4 a_{2}}\right) / 2, K_{2}=\left(a_{1}+\sqrt{a_{1}^{2}-4 a_{2}}\right) / 2$, $a_{1}=k_{01} c+k_{10}+k_{12}, a_{2}=c k_{01} k_{12}, C_{1}=-K_{2} /\left(K_{2}-K_{1}\right)$, and $C_{2}=K_{1} /\left(K_{2}-K_{1}\right)$. According to Eq. (3), the time dependence of the probability function for each state is given by a sum of two exponents with different decay times. The general solution (3) of the MichaelisMenten equations (2) remains valid for all values of the reaction rate constants and any dependence of the rate constants on applied force.

Consider the average time $\tau$ taking the system to reach state 2 . It defines the timescale for the bond dissociation process. To find an expression for $\tau$, consider the probability that the system is not in state 2, i.e., $1-p_{2}(t)$. The corresponding probability density $P(t)$ is given by

$$
\begin{aligned}
P(t) & =\left(1-p_{2}(t)\right) / \int_{0}^{\infty}\left(1-p_{2}(t)\right) d t \\
& =\frac{C_{1} \exp \left(-K_{1} t\right)+C_{2} \exp \left(-K_{2} t\right)}{C_{1} / K_{1}+C_{2} / K_{2}} .
\end{aligned}
$$

Then, $\tau$ can be easily found from

$$
\tau=\int_{0}^{\infty} t P(t) d t=\frac{C_{1} K_{2}^{2}+C_{2} K_{1}^{2}}{K_{1} K_{2}\left(C_{1} K_{2}+C_{2} K_{1}\right)}
$$

As pointed out by Brujic et al. ${ }^{6}$ the probability that $n$ domains in an $N$ domain chain will be unfolded is related to the unfolding probability for an isolated domain by the binomial distribution

$$
P(N, n, t)=\frac{N !}{n !(N-n) !} p_{2}(t)^{n}\left(1-p_{2}(t)\right)^{N-n},
$$

where $n=0,1,2, \ldots, N$. In the present case, $p_{2}(t)$ is given by Eq. (3). According to the binomial distribution (6), the average number of unfolded domains is equal to

$$
\mu(t)=\sum_{n=0}^{N} n P(N, n, t)=N p_{2}(t),
$$

and the mean-square deviation of this number from the average is given by

$$
\sigma(t)^{2}=\sum_{n=0}^{N}(n-\mu(t))^{2} P(N, n, t)=N p_{2}(t)\left(1-p_{2}(t)\right) .
$$

If $l$ denotes the length of one unfolded domain, then in accordance with Eq. (7), the chain extension averaged over an ensemble of experiments $L(t)$ is equal to

$$
L(t)=l N p_{2}(t) .
$$

The corresponding value can be written including the standard deviation from the average as

$$
L(t)^{ \pm}=l\left[N p_{2}(t) \pm \sqrt{N p_{2}(t)\left(1-p_{2}(t)\right)}\right] .
$$

Since the chain extension, Eq. (9), is directly proportional to the bond dissociation probability $p_{2}(t)$, the average timescale for the full extension of the chain is equal to the average timescale for the bond dissociation, Eq. (5). Therefore, a study of the time-dependent extension of a chain composed of many domains generates directly the lifetime of a single disulfide bond within the chain.

A number of expressions useful for the analysis of the experimental data can be derived from Eq. (6). For instance, the time-dependent probability density to have $n$ unfolded domains can be computed as

$$
P_{n}(t)=p_{2}(t)^{n}\left(1-p_{2}(t)\right)^{N-n} / \int_{0}^{\infty} p_{2}(t)^{n}\left(1-p_{2}(t)\right)^{N-n} d t .
$$

This formula can be used to obtain the average time required for the unfolding of any $n$ domains:

$$
\tau^{(n)}=\int_{0}^{\infty} t P_{n}(t) d t
$$

\section{APPROXIMATION TO THE ENZYMATIC UNFOLDING MODEL}

The expressions derived in the previous section can be used directly to analyze the experimental results. ${ }^{30}$ Such analysis can be greatly facilitated, however, by the approximations derived in this section. The experimental data indicate that the strength of the applied force and the enzyme concentration strongly influence each other, see Fig. 2c of Wiita et al. ${ }^{30}$ and, therefore, should enter the rate expression as a product. In order for Eq. (2) to satisfy this condition, the transition from the intermediate state 1 to the broken bond state 2 should be significantly faster than all other timescales in the system. Therefore, $k_{12} \gg k_{01} c$; $k_{10}$, leading to the conclusion that the dimensionless ratio $a_{2} / a_{1}^{2}$ in Eq. (3) must be small, i.e., $a_{2} / a_{1}^{2} \ll 1$. This condition allows us to write down the following approximate relationships

$$
\begin{aligned}
& K_{1} \approx a_{2} / a_{1}, \quad K_{2} \approx a_{1}-a_{2} / a_{1}, \quad C_{1} \approx-1-a_{2} / a_{1}^{2}, \\
& C_{2} \approx a_{2} / a_{1}^{2} .
\end{aligned}
$$


Considering the rate terms in Eqs. (3) and (4), we conclude that one of the decay times is short, $1 / K_{2} \approx 1 / k_{12}$, while the other decay time is long, $1 / K_{1} \approx 1 / c k_{01}$. Hence, if one is not interested in the very short-time behavior of the state populations and focuses on the long-time properties of the probabilities, Eq. (3) can be simplified to

$$
p_{2}(t) \approx 1-\exp \left[-c k_{01}(f) t\right]
$$

in the zeroth order approximation with respect to the small dimensionless parameter $a_{2} / a_{1}^{2}$. Note that Eq. (13) is already the first-order approximation. Equation (14) implies that at the simplest level the bond dissociation process is determined by the rate of the first step in the Michaelis-Menten mechanism. This first step is associated with the first barrier in the potential energy profile shown in Fig. 1. Therefore, the second barrier and the intermediate state can be dropped from consideration, and the reaction profile can be approximated as shown by the dashed line in Fig. 1. The resulting 2-state approximation is significantly simpler than the original 3 -state scheme ${ }^{30}$ as well as the 4 -state scheme proposed more recently. ${ }^{31}$

The rate constant entering Eq. (14) depends on the applied force. The force dependence will be described according to the deformation model ${ }^{18}$ that associates the force-induced changes in the bond lifetime with conformational changes within the protein system. In the present system, these changes involve rearrangements and displacements of the disulfide group relative to the surrounding atoms of the I27 domain and the Trx enzyme. Prior to considering an explicit functional form of the force dependence of the rate constant, we need to re-write some of the general results obtained in the previous section in view of the approximation given by Eq. (14). In particular, Eq. (5) for the bond lifetime becomes

$$
\tau(f)=1 / c k_{01}(f) .
$$

The bond lifetime determines the timescale for the extension of the I27 domain chain. According to Eq. (12), the timescale $\tau^{(0)}$ on which none of the disulfide bonds in the $N=8$-domain chain are broken, yet is shorter than the lifetime of an isolated bond by a factor of 8 :

$$
\tau^{(0)}(f)=1 / 8 c k_{01}(f) .
$$

In contrast, the time needed to unfold 7 out of the 8 domains is greater than the isolated bond lifetime, Eq. (15), by nearly three times: $\tau^{(7)} \approx 2.7 \tau(f)$.

The zeroth-order approximation described in this section works very well and captures the most essential features of the experimental data. In this approximation, the disulfide bond dissociation rate

$$
r_{0}(f)=c k_{01}(f)
$$

shows linear dependence on the enzyme concentration c. Note that Eq. (17) is the inverse of Eq. (15) for the bond lifetime. To reproduce the mild nonlinearities in the concentration dependence of the rate constants detected experimentally, one can use the first-order approximation

$$
r_{1}(f)=c k_{01}(f) /\left[1+k_{10} / k_{12}+c k_{01}(f) / k_{12}\right]
$$

obtained from Eq. (5) using Eq. (13). Note that the only explicit force dependence considered in Eq. (18) is that of $k_{01}(f)$.

\section{DEFORMATION MODEL OF ENZYME CATALYSIS IN THE PRESENCE OF FORCE}

The one-dimensional reaction profile shown in Fig. 1 is a great simplification. It is a projection of a multidimensional motion of the complex system comprising the disulfide bond itself, substrate, enzyme, and solvent. Relative displacements of atoms and largerscale components such as residues or protein domains can change the shape of the interaction potential. For instance, it is exactly structural rearrangements of this kind that facilitate biological receptor/ligand interactions and form the basis for the induced receptor/ligand fitting idea. ${ }^{14}$ Similar effects can be expected when proteins are subjected to mechanical forces. Residues that come in contact inside the binding pocket can shift, thereby improving or weakening receptor/ligand binding. The effect of the force-induced deformation of the interaction energy profile was considered in Pereverzev and Prezhdo. ${ }^{18}$ The deformation effect acts in parallel with the Bell renormalization of the barrier height by applied force. ${ }^{4}$ The Bell term is commonly used to describe the effect of the applied force on biological bonds, the majority of which are slip-bonds. It should be emphasized that the deformation and Bell effects are qualitatively different. The Bell term describes the force-induced modification in the receptor-ligand interaction assuming rigid receptor and ligand. On the other hand, the deformation term represents conformational changes that take place within the receptorligand pair due to the applied force and alter the interaction potential.

Molecular dynamics simulations show ${ }^{30}$ that binding of the Trx enzyme to the I27 domain containing the disulfide bond is not optimal for the disulfide dissociation reaction. The Trx groove forms a $70^{\circ}$ angle with respect to the bond. At the same time, the Trx catalysis proceeds by the $S_{N} 2$ reaction mechanism, ${ }^{11,12}$ which requires that the disulfide bond and the sulfur atom of the enzyme align in the same direction forming a 
straight line. ${ }^{15,25,29,30}$ Therefore, in order for the $S_{N} 2$ reaction to proceed, the system must overcome a deformation energy barrier associated with the rotation of the disulfide bond with respect to the Trx grove. Simulations show ${ }^{30}$ that the bond rotation causes a contraction of the I27 domain, and that the contraction is opposed by the pulling force. Under these conditions, the force increases the deformation energy barrier, which can be represented by

$$
\Delta E_{\mathrm{d}}(f)=\alpha\left[1-\exp \left(-f / f_{0}\right)\right]
$$

as shown in Pereverzev and Prezhdo. ${ }^{18}$ Here, $\alpha$ is the additional deformation energy that must be overcome to align the disulfide bond and the Trx groove in the presence of force $f$, and $f_{0}$ is the force above which the properties of the bond do not change significantly any more. As argued in Pereverzev and Prezhdo, ${ }^{18}$ the functional form of Eq. (19) is chosen to ensure that the deformation energy grows linearly with applied force at small forces, and that the deformation saturates, such that further force increase beyond $f_{0}$ has little effect on the bond/groove alignment. The deformation energy $\alpha$ is positive in the present case, $\alpha>0$, since the force raises the energy barrier. It can be negative in other situations. ${ }^{18}$

The current interpretation of the deformation effect is based on the atomistic simulations ${ }^{30}$ and is associated with the rotation of the disulfide bond with respect to the Trx groove causing the I 27 substrate to contract. The strong correlation between the force and enzyme concentration apparent in the experimental data, see Fig. 2c of Wiita et al., ${ }^{30}$ suggests that the deformation occurs during the transition from the initial to the intermediate state, Eqs. (2). This step involves binding of the enzyme to the substrate. It is feasible, therefore, that the force-induced deformation involves not only the bond rotation but also a larger scale conformational change of the substrate, affecting the enzyme-substrate affinity.

In addition to opposing the conformational changes required to align the disulfide bond with respect to the Trx groove, the applied force acts to lower the barrier leading toward the transition state of the $S_{N} 2$ reaction catalyzed by the Trx enzyme. By interacting with Trx, the disulfide bond is significantly weakened and a new bond involving the sulfur atom of the enzyme is starting to form. The force-induced change in the $S_{N} 2$ reaction barrier can be described by the traditional term first introduced by Bell ${ }^{4}$

$$
\Delta E_{\mathrm{B}}(f)=-x_{01} f
$$

where $x_{01}$ is the barrier width. The two contributions to the energy barrier separating states 0 and 1, Eqs. (19) and (20), emphasize the fact that the force dependence of the barrier is determined by two independent parameters - the bond/grove alignment angle and the displacement of the sulfur atoms with respect to each other.

Combining Eqs. (19) and (20), the overall dependence of the reaction rate constant of the applied force can be written in the form

$$
k_{01}(f)=k_{01}^{0} \exp \left(-\frac{\Delta E_{\mathrm{d}}(f)+\Delta E_{\mathrm{B}}(f)}{k_{\mathrm{B}} T}\right),
$$

where $k_{01}^{0}$ is the rate constant in the absence of force, $k_{\mathrm{B}}$ is the Boltzmann constant, and $T$ is temperature. The deformation and Bell terms in Eq. (21) describe the catch and slip behavior associated with increasing and then decreasing lifetime with increasing force. According to Eqs. (19)-(21) the deformation model of the catch-slip force dependence of the rate constant contains four parameters. They are the force-free rate constant $k_{01}^{0}$, the barrier width $x_{01}$, the deformation energy $\alpha$, and the saturation force $f_{0}$. It follows from Eqs. (19)-(21) that if $\alpha>x_{01} f_{0}$, the rate constant (21) is minimized by the force

$$
f_{\mathrm{m}}=f_{0} \ln \frac{\alpha}{x_{01} f_{0}} .
$$

The ratio of the maximum bond lifetime generated by $f_{\mathrm{m}}$ to the lifetime at zero force defines the catchbinding efficiency ${ }^{21}$

$$
\frac{\tau\left(f_{\mathrm{m}}\right)}{\tau(0)}=\exp \left[\frac{\alpha-x_{01}\left(f_{0}+f_{\mathrm{m}}\right)}{k_{\mathrm{B}} T}\right] .
$$

The efficiency can significantly exceed one, if the deformation energy is greater than the Bell term $\alpha>x_{01}\left(f_{0}+f_{\mathrm{m}}\right)$. An alternative definition of the catch-slip efficiency associated with the presence of both a maximum and a minimum in the force-dependent bond lifetime is given in Beste and Hammer. ${ }^{5}$

The deformation model presented above has been successfully used to describe the anomalous dependence of the bond lifetime on the applied force in the P,L-selectins/PSGL- ${ }^{18}$ and actin/myosin ${ }^{22}$ catch bonds.

The force dependence of the reaction rate coefficient given by Eq. (21) is used below to describe the anomalous force dependence of the disulfide bond dissociation catalyzed by the Trx enzyme. ${ }^{30}$ In contrast to the model used in Wiita et al. ${ }^{30}$ the proposed deformation model contains only one bond dissociation pathway. The key force dependence enters the reaction rate $k_{01}(f)$ of the first step in the Michaelis-Menten mechanism, Eq. (2).

\section{DISCUSSION OF THE EXPERIMENTAL DATA}

The anomalous force dependence of the I27 chain extension time is a particularly interesting 
experimental finding reported by Wiita et $a l .^{30}$ According to our model, the measured extension timescale characterizes the lifetime of a single disulfide bond, Eqs. (5) and (15). Figures 2a, 2b, and 2c shows the experimental dependence of the bond dissociation rate on force $r(f)$ for three Trx enzymes, including wild type Trx, $\operatorname{Trx}(\mathrm{P} 34 \mathrm{H})$ mutant, and human Trx, respectively. The red dots present the experimental data, while the solid lines give the theoretical results, obtained within the simplest approximation $r_{0}(f)$ given
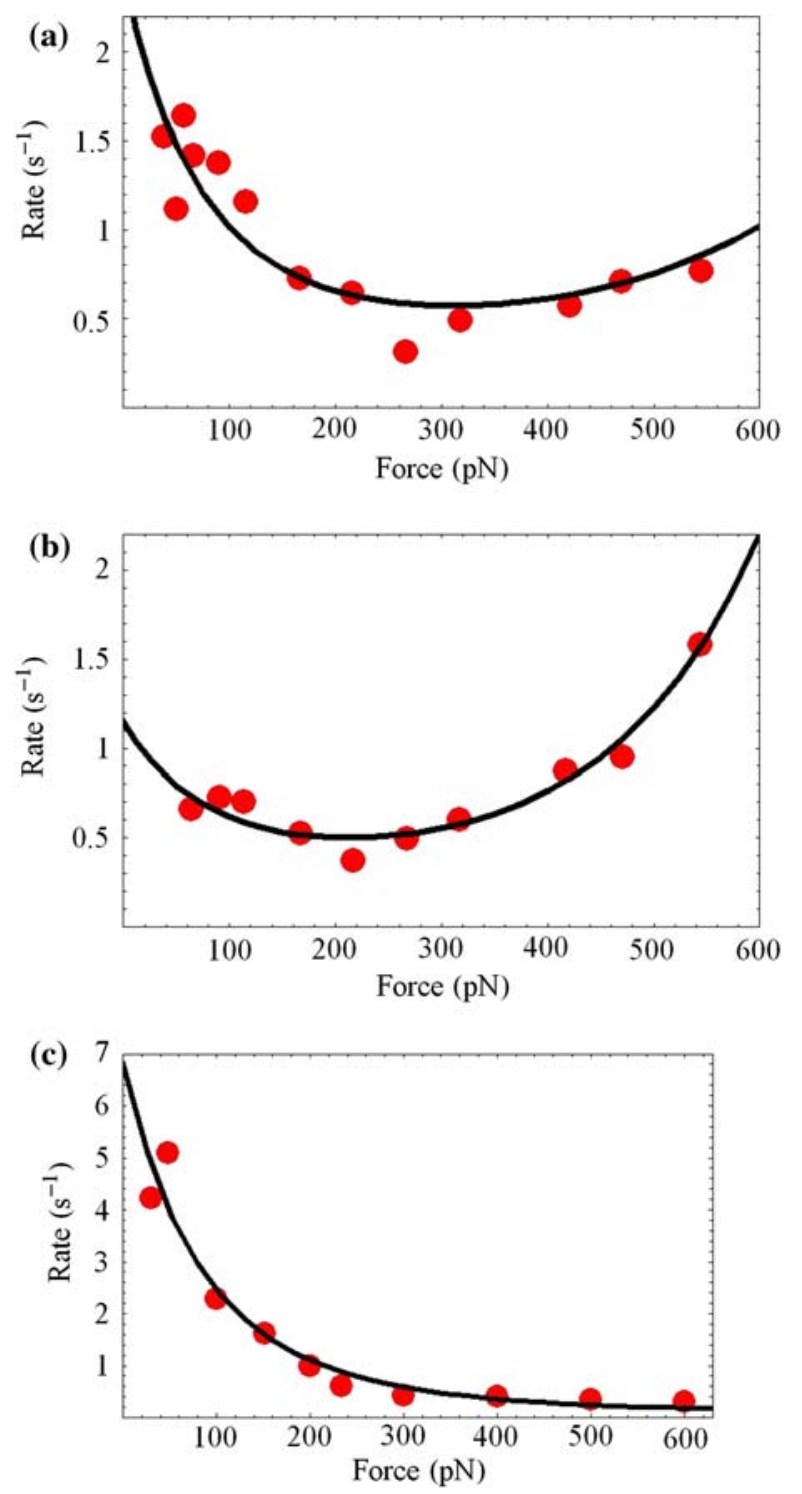

FIGURE 2. Dependence of the disulfide bond dissociation rate constant on applied force. The experimental data are shown by red points, and the theoretical results are given by black lines. Three different Trx enzymes are considered: (a) WT Trx with concentration $c=8 \mu \mathrm{M}$, (b) Trx (P34H), $c=8 \mu \mathrm{M}$, and (c) Human Trx, $c=10 \mu \mathrm{M}$. The parameters of the theoretical model are (a) $k_{01}^{0}=0.27 \mu \mathrm{M}^{-1} \mathrm{~s}^{-1}, \alpha=204.8 \mathrm{pNA}, f_{0}=$ $289.1 \mathrm{pN}, x_{01}=0.25 \AA$; (b) $k_{01}^{0}=0.12 \mu \mathrm{M}^{-1} \mathrm{~s}^{-1}, \alpha=183.6 \mathrm{pNA}$ $f_{0}=300 \mathrm{pN}, \quad x_{01}=0.31 \AA ; \quad$ and (c) $k_{01}^{0}=0.68 \mu \mathrm{M}^{-1} \mathrm{~s}^{-1}$, $\alpha=261.1 \mathrm{pNA}, f_{0}=500 \mathrm{pN}, x_{01}=0.06 \AA$. by Eq. (17) together with Eqs. (19)-(21). The four model parameters, which have been defined following Eq. (21), are presented in the figure caption for each enzyme type.

The catch-binding efficiencies, Eq. (23), are equal to 4 for WT Trx and 2 for $\operatorname{Trx}(\mathrm{P} 34 \mathrm{H})$. The efficiency is much higher for Human Trx, reaching nearly 60. According to Eqs. (22) and (23) the efficiency does not depend on the enzyme concentration, which is slightly higher for Humant Trx, $c=10 \mu \mathrm{M}$, than for WT Trx and $\operatorname{Trx}(\mathrm{P} 34 \mathrm{H}), c=8 \mu \mathrm{M}$. Human Trx exhibits high catch-binding efficiency due to the large $\alpha$ and very small $x_{01}$, see caption to Fig. 2 . The disulfide bond dissociation is slowed down the most by the following forces, $f_{\mathrm{m}}=301,204$, and $1085 \mathrm{pN}$ for WT Trx, $\operatorname{Trx}(\mathrm{P} 34 \mathrm{H})$, and Human Trx, respectively. The data shown in Fig. $2 \mathrm{c}$ are limited to $f<f_{\mathrm{m}}$ and, therefore, the rate constant only decreases within this force range.

The analysis shows that the disulfide bond catalyzed by Human Trx is the most efficient catch system of the three Trx examples considered by Wiita et al. ${ }^{30}$ The Human Trx system exhibits the largest deformation energy $\alpha \approx 261 \mathrm{pNA}$, and the deformation saturates by the largest force $f_{0}=500 \mathrm{pN}$. The deformation energy of $\alpha \approx 261 \mathrm{pN} \AA$ corresponds to about $6 k_{\mathrm{B}} T$ at room temperature. The ratio of the deformation energy to the saturating force, $\Delta x=\alpha / f_{0}$, characterize the forceinduced deformation distance. According to the current interpretation, in which the deformation is associated with the rotation of the disulfide bond and causes contraction of the I27 domain, this distance quantifies the contraction. The contraction distances are $0.71,0.6$, and $0.52 \AA$ for WT Trx, Trx $(\mathrm{P} 34 \mathrm{H})$, and Human Trx, respectively. These magnitudes are quite reasonable, since they originate from rotation of a single chemical bond by approximately a $70^{\circ}$ angle. ${ }^{30}$ The most efficient catch system involving Human Trx is the stiffest one. Its contraction is the shortest, and requires the largest force and the most amount of energy.

The distances $x_{01}$ characterizing the width of the barrier for the $S_{N} 2$ reaction are shorter than the contraction distances discussed above, see caption to Fig. 2. This should be expected, since dissociation of a chemical bond involves very small displacements of atoms. The $S_{N} 2$ distance is the shortest for the Human Trx system, $x_{01}=0.06 \AA$. Because it is so small, the Bell-type contribution, Eq. (20), to the rate constant, Eq. (21), becomes significant only at large forces. As a result, the catch-slip transition in the Human Trx system occurs at a very large force, $f_{\mathrm{m}}=1085 \mathrm{pN}$. At smaller forces, the Bell term is not able to suppress the deformation term, Eq. (19), responsible for the catchbehavior. This factor contributes to the large catchefficiency of the Human Trx system, $\tau\left(f_{\mathrm{m}}\right) / \tau(0) \approx 60$, Eq. (23). 


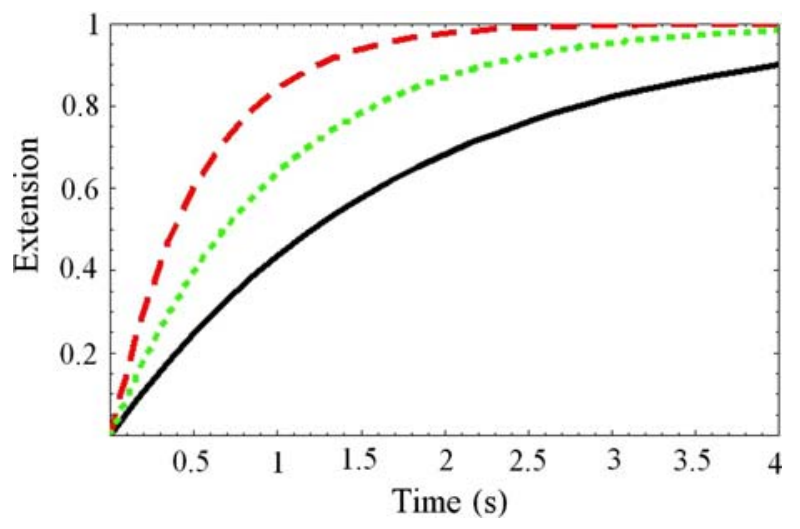

FIGURE 3. Relative extension of the 8-domain protein chain catalyzed by WT Trx with concentration $c=8 \mu \mathrm{M}$ as a function of time for three force values: $25 \mathrm{pN}$ (red dashes), $300 \mathrm{pN}$ (black solid line), and $600 \mathrm{pN}$ (green dots). The model parameters are the same as in Fig. 2a.

Equation (9) relates the dissociation probability for a single disulfide bond, $p_{2}(t)$, to the extension $L(t)$ of the chain of $N$ segments, each containing a bond. Figure 3 shows the evolution of the relative extension of the 8-domain chain catalyzed by WT Trx. The chain extension data shown in Fig. 3 were obtained using Eqs. (9), (14), and (21) with the disulfide bond dissociation rate parameters and WT Trx concentration given in the caption to Fig. 2a. Three typical force values are considered, $f=25,300$, and $600 \mathrm{pN}$. The theoretical curves agree well with the corresponding experimental measurements, see Fig. 2a of Wiita et al. The most efficient catch binding occurs in this WT Trx system at the intermediate force $f=300 \mathrm{pN}$, and the chain extension takes the most time. The extension proceeds much more rapidly at both small and large forces, $f=25$ and $600 \mathrm{pN}$.

In the simplest description of the enzyme-catalyzed dissociation of the disulfide bond, the dissociation rate constant is a linear function of the enzyme concentration, Eq. (17). However, the experiments ${ }^{30}$ show modest deviations from the linearity. According to the more accurate approximation, Eq. (18), to the full Michaelis-Menten mechanism, Eq. (2), the deviations occur due to the contribution of the intermediate state 1, see Fig. 1. The linear regime described by Eq. (17) makes the assumption that state 1 is short lived. Figure 4 shows the experimental data (blue points) and theoretical results (lines) for the disulfide bond dissociation rate constant in the WT Trx system subjected to the $25 \mathrm{pN}$ force. The dashed line gives the linear concentration dependence predicted by Eq. (17), while the solid line includes the nonlinearity, Eq. (18). The additional parameters entering Eq. (18) are $k_{10} /$ $k_{12}=0.01$ and $k_{12}=20 \mathrm{~s}^{-1}$. These rate constants are associated with transitions from the intermediate state

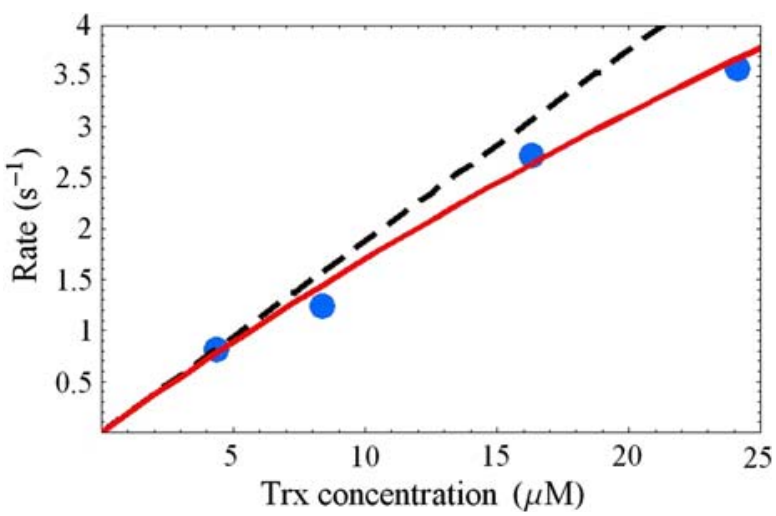

FIGURE 4. Concentration dependence of the disulfide bond dissociation rate. The blue points give the experimental data. The solid and dashed lines show the theoretical results with and without the intermediate state, Eqs. (18) and (17), respectively. The parameters are the same as in Fig. 2a. The additional parameters associated with the intermediate state are $k_{10} / k_{12}=0.01 ; k_{12}=20 \mathrm{~s}^{-1}$.

1 to the initial state 0 and final state 2 , and are taken to be independent of force. All other parameters are the same as in Fig. 2a. The deviation from linearity gives the following value of the small dimensionless parameter that was used to obtain Eq. (13), $a_{2} / a_{1}^{2} \approx$ 0.1. The dashed line in Fig. 4 assumes that it is zero. The solid line is the first-order correction with respect to this parameter.

\section{CONCLUSIONS}

The anomalous dependence of the disulfide bond lifetime on the strength of the applied force has been described using a single bond dissociation pathway, in contrast to the two-pathway model proposed in the original publication. ${ }^{30}$ It has been shown for the developed model that the measured timescale of the forced extension of the 8-domain protein chain directly gives the lifetime of a single disulfide bond contained in each domain. The model has been successfully applied to three experimentally studied systems involving WT Trx, Trx (P34H), and Human Trx enzymes.

The proposed model is based on the usual MichaelisMenten scheme of enzyme catalysis, involving the initial, final, and intermediate states. The exact solution to the kinetic equations of the Michaelis-Menten scheme has been presented. Application of the scheme to the experimental data relies on two assumptions. First, the transition from the intermediate to the final state is significantly faster than all other transitions. This assumption is not required, but it simplifies the analysis and gives good agreement between theory and experiment. Second, the rate coefficient for the transition from the initial to the intermediate state 
exhibits anomalous dependence on the applied force, while in model $^{30}$ this rate coefficient had no force dependence. Our assumptions are justified by the properties of the experimental data, in particular, by the fact that the effects of the force and enzyme concentration on the reaction rate are strongly correlated. The assumptions lead to the conclusion that the intermediate state plays little role in the observed force dependence of the disulfide bond dissociation catalyzed by Trx.

The deformation mode ${ }^{18}$ for the anomalous force dependence of the enzymatic dissociation of the disulfide bond incorporates two independent effects. First, the force increases the energy barrier associated with the rotation of the disulfide bond and substrate shortening. This bond deformation effect saturates at some force, Eq. (19). Second, the barrier leading to the transition state of the $S_{N} 2$ reaction is lowered by the force according to the traditional Bell expression, Eq. (20). The atom displacements involved in the $S_{N} 2$ reaction occur on a smaller scale than the bond rotation and substrate shortening. These two independent effects determine the force-induced change in the height of the barrier leading from the initial to the intermediate state, Fig. 1. The deformation energy parameter $\alpha$ characterizing this process is a key characteristic of the catch-slip transition. The largest deformation energy obtained for Human Trx is on the order of $6 k_{\mathrm{B}} T$. Alternative estimates of the deformation energy that can be produced by independent experimental measurements or computer simulation would be extremely valuable for understanding the enzyme-catalyzed chemical catch-binding.

In the simplest approximation based on the small ratio of all other rate constants to the rate constant for the transition from the intermediate to the final state, the three-state Michaelis-Menten scheme simplifies to a two-state scheme, and the experimental data can be described using only four independent parameters, Eq. (21). These are the dissociation rate constant in the absence of force, the width of the $S_{N} 2$ reaction barrier, the force-induced bond deformation energy, and the force saturating this deformation. The simple 4-parameter picture provided by the deformation model gives good agreement with the experimental data on disulfide bond dissociation catalyzed by three different enzymes: WT Trx, Trx (P34H), and Human Trx. The small deviations from the linear dependence of the bond dissociation rate on the Trx concentration require a higher-order approximation to the full Michaelis-Menten scheme and involve two additional constants describing escape from the intermediate state. We would like to emphasize that the description of the catch-slip anomaly proposed in the current work is not unique, and that several alternative models are available in the literature. . $^{2,16,21,26,31}$ The current model differs from the alternative interpretations by its mathematical simplicity and by the bond deformation concept that is used to rationalize the physics of catchbinding.

The Trx-catalyzed disulfide bond presents the first example of a chemical catch-bond. The strongest form of catch-binding is observed with the Human Trx, leading to the catch-binding efficiency on the order of 60 .

\section{ACKNOWLEDGMENTS}

The authors thank Evgeni Sokurenko for multiple and fruitful discussions. Financial support of National Science Foundation grant CHE-0701517, National Institute of Health grant NIH R01 AI50940, and Petroleum Research Fund of the American Chemical Society grant 46772-AC6 is gratefully acknowledged.

\section{OPEN ACCESS}

This article is distributed under the terms of the Creative Commons Attribution Noncommercial License which permits any noncommercial use, distribution, and reproduction in any medium, provided the original author(s) and source are credited.

\section{REFERENCES}

${ }^{1}$ Aprikian, P., V. Tchesnokova, B. Kidd, O. Yakovenko, V. Yarov-Yarovoy, E. Trinchina, V. Vogel, W. Thomas, and E. Sokurenko. Interdomain interaction in the FimH adhesin of Escherichia coli regulates the affinity to mannose. J. Biol. Chem. 282(32):23437-23446, 2007.

${ }^{2}$ Barsegov, V., and D. Thirumalai. Dynamics of unbinding of cell adhesion molecules: transition from catch to slip bonds. Proc. Natl Acad. Sci. USA 102(6):1835-1839, 2005. ${ }^{3}$ Bartolo, D., I. Derenyi, and A. Ajdari. Dynamic response of adhesion complexes: beyond the single-path picture. Phys. Rev. E 65(5 Pt 1):051910, 2002.

${ }^{4}$ Bell, G. I. Models for specific adhesion of cells to cells. Science 200(4342):618-627, 1978.

${ }^{5}$ Beste, M. T., and D. A. Hammer. Selectin catch-slip kinetics encode shear threshold adhesive behavior of rolling leukocytes. Proc. Natl. Acad. Sci. USA 105(52):2071620721, 2008.

${ }^{6}$ Brujic, J., R. I. Hermans, S. Garcia-Manyes, K. A. Walther, and J. M. Fernandez. Dwell-time distribution analysis of polyprotein unfolding using force-clamp spectroscopy. Biophys. J. 92(8):2896-2903, 2007.

${ }^{7}$ Dembo, M., D. C. Torney, K. Saxman, and D. Hammer. The reaction-limited kinetics of membrane-to-surface adhesion and detachment. Proc. R. Soc. Lond. B 234(1274): 55-83, 1988.

${ }^{8}$ Evans, E., A. Leung, V. Heinrich, and C. Zhu. Mechanical switching and coupling between two dissociation pathways 
in a P-selectin adhesion bond. Proc. Natl Acad. Sci. USA 101(31):11281-11286, 2004.

${ }^{9}$ Gunnerson, K. N., Y. V. Pereverzev, and O. V. Prezhdo. Atomistic simulations combined with analytic theory to study the response of the P-selectin/PSGL-1 complex to an external force. J. Phys. Chem. B 113(7):2090-2100, 2009.

${ }^{10}$ Guo, B., and W. H. Guilford. Mechanics of actomyosin bonds in different nucleotide states are tuned to muscle contraction. Proc. Natl Acad. Sci. USA 103(26):9844-9849, 2006.

${ }^{11}$ Holmgren, A. Thioredoxin. Annu. Rev. Biochem. 54:237271, 1985.

${ }^{12}$ Holmgren, A. Thioredoxin structure and mechanism: conformational changes on oxidation of the active-site sulfhydryls to a disulfide. Structure 3(3):239-243, 1995.

${ }^{13}$ Kolomeisky, A. B. Channel-facilitated molecular transport across membranes: attraction, repulsion, and asymmetry. Phys. Rev. Lett. 98(4):4, 2007.

${ }^{14}$ Koshland, D. E. The key-lock theory and the induced fit theory. Angew. Chem. Int. Ed. 33(23-24):2375-2378, 1994.

${ }^{15}$ Koti Ainavarapu, S. R., A. P. Wiita, L. Dougan, E. Uggerud, and J. M. Fernandez. Single-molecule force spectroscopy measurements of bond elongation during a bimolecular reaction. J. Am. Chem. Soc. 130(20):64796487, 2008.

${ }^{16}$ Lou, J. Z., and C. Zhu. A structure-based sliding-rebinding mechanism for catch bonds. Biophys. J. 92(5):1471-1485, 2007.

${ }^{17}$ Marshall, B. T., M. Long, J. W. Piper, T. Yago, R. P. McEver, and C. Zhu. Direct observation of catch bonds involving cell-adhesion molecules. Nature 423(6936):190 193, 2003.

${ }^{18}$ Pereverzev, Y. V., and O. V. Prezhdo. Force-induced deformations and stability of biological bonds. Phys. Rev. E 73:050902, 2006.

${ }^{19}$ Pereverzev, Y. V., and O. V. Prezhdo. Dissociation of biological catch-bond by periodic perturbation. Biophys. J. 91(2):L19-L21, 2006.

${ }^{20}$ Pereverzev, Y. V., and O. V. Prezhdo. Universal laws in the force-induced unraveling of biological bonds. Phys. Rev. E 75(1 Pt 1):011905, 2007.

${ }^{21}$ Pereverzev, Y. V., O. V. Prezhdo, M. Forero, E. V. Sokurenko, and W. E. Thomas. The two-pathway model for the catch-slip transition in biological adhesion. Biophys. J. 89(3):1446-1454, 2005.

${ }^{22}$ Pereverzev, Y. V., O. V. Prezhdo, and E. V. Sokurenko. Anomalously increased lifetimes of biological complexes at zero force due to the protein-water interface. J. Phys. Chem. B 112(36):11440-11445, 2008.

${ }^{23}$ Pereverzev, Y. V., O. V. Prezhdo, W. E. Thomas, and E. V. Sokurenko. Distinctive features of the biological catch bond in the jump-ramp force regime predicted by the two-pathway model. Phys. Rev. E 72(1):010903, 2005.

${ }^{24}$ Sarangapani, K. K., T. Yago, A. G. Klopocki, M. B. Lawrence, C. B. Fieger, S. D. Rosen, R. P. McEver, and C. Zhu. Low force decelerates L-selectin dissociation from P-selectin glycoprotein ligand-1 and endoglycan. J. Biol. Chem. 279(3):2291-2298, 2004.

${ }^{25}$ Szoszkiewicz, R., S. R. Ainavarapu, A. P. Wiita, R. PerezJimenez, J. M. Sanchez-Ruiz, and J. M. Fernandez. Dwell time analysis of a single-molecule mechanochemical reaction. Langmuir 24(4):1356-1364, 2008.

${ }^{26}$ Thomas, W., M. Forero, O. Yakovenko, L. Nilsson, P. Vicini, and E. Sokurenko. Catch-bond model derived from allostery explains force-activated bacterial adhesion. Biophys. J. 90(3):753-764, 2006.

${ }^{27}$ Thomas, W. E., E. Trintchina, M. Forero, V. Vogel, and E. V. Sokurenko. Bacterial adhesion to target cells enhanced by shear force. Cell 109(7):913-923, 2002.

${ }^{28}$ Thomas, W. E., V. Vogel, and E. Sokurenko. Biophysics of catch bonds. Annu. Rev. Biophys. 37:399-416, 2008.

${ }^{29}$ Wiita, A. P., S. R. Ainavarapu, H. H. Huang, and J. M. Fernandez. Force-dependent chemical kinetics of disulfide bond reduction observed with single-molecule techniques. Proc. Natl Acad. Sci. USA 103(19):7222-7227, 2006.

${ }^{30}$ Wiita, A. P., R. Perez-Jimenez, K. A. Walther, F. Grater, B. J. Berne, A. Holmgren, J. M. Sanchez-Ruiz, and J. M. Fernandez. Probing the chemistry of thioredoxin catalysis with force. Nature 450(7166):124-127, 2007.

${ }^{31}$ Xue, X. C., L. C. Gong, F. Liu, and Z. C. Ou-Yang. Two-pathway four-state kinetic model of thioredoxincatalyzed reduction of single forced disulfide bonds. Phys. Rev. E 77(5 Pt 1):050903, 2008.

${ }^{32}$ Zhu, C., J. Z. Lou, and R. P. McEver. Catch bonds: physical models, structural bases, biological function and rheological relevance. Biorheology 42(6):443-462, 2005. 